\title{
Focal Therapy in Prostate Cancer- Report from a Consensus Panel
}

\author{
J. de la Rosette, M.D., H. Ahmed, M.D., ${ }^{2}$ J. Barentsz, M.D., ${ }^{3}$ T. Bjerklund Johansen, M.D., ${ }^{4}$ M. Brausi, M.D., ${ }^{5}$ \\ M. Emberton, M.D., ${ }^{2}$ F. Frauscher, M.D., ${ }^{6}$ D. Greene, M.D., ${ }^{7}$ M. Harisinghani, M.D., ${ }^{8}$ K. Haustermans, M.D., 9 \\ A. Heidenreich, M.D., ${ }^{10}$ G. Kovacs, M.D., ${ }^{11}$ M. Mason, M.D., ${ }^{12}$ R. Montironi, M.D., ${ }^{13}$ V. Mouraviev, M.D., ${ }^{14}$ \\ T. de Reijke, M.D., S. Taneja, M.D., ${ }^{15}$ S. Thuroff, M.D., ${ }^{16}$ B. Tombal, M.D., ${ }^{17}$ \\ J. Trachtenberg, M.D., ${ }^{18}$ H. Wijkstra, M.D., and T. Polascik, M.D. ${ }^{14}$
}

\begin{abstract}
Purpose: To establish a consensus in relation to case selection, conduct of therapy, and outcomes that are associated with focal therapy for men with localized prostate cancer.

Material and Methods: Urologic surgeons, radiation oncologists, radiologists, and histopathologists from North America and Europe participated in a consensus workshop on focal therapy for prostate cancer. The consensus process was face to face within a structured meeting, in which pertinent clinical issues were raised, discussed, and agreement sought. Where no agreement was possible, this was acknowledged, and the nature of the disagreement noted.

Results: Candidates for focal treatment should have unilateral low- to intermediate-risk disease with clinical stage $\leq \mathrm{cT}_{2 \mathrm{a}}$. Prostate size and both tumor volume and tumor topography are important case selection criteria that depend on the ablative technology used. Currently, the best method to ascertain the key characteristics for men who are considering focal therapy is exposure to transperineal template mapping biopsies. MRI of the prostate using novel techniques such as dynamic contrast enhancement and diffusion weighed imaging are increasingly being used to diagnose and stage primary prostate cancer with excellent results. For general use, however, these new techniques require validation in prospective clinical trials. Until such are performed, MRI will, in most centers, continue to be an investigative tool in assessing eligibility of patients for focal therapy. Conclusions: Consensus was derived for most of the key aspects of case selection, conduct of treatment, and outcome measures for men who are undergoing focal therapy for localized prostate cancer. The level of agreement achieved will pave the way for future collaborative trials.
\end{abstract}

\footnotetext{
${ }^{1}$ Department of Urology, AMC University Hospital, Amsterdam, The Netherlands.

${ }^{2}$ Division of Surgery and Interventional Science, UCLH/UCL Comprehensive Biomedical Centre, London, United Kingdom.

${ }^{3}$ Department of Radiology, UMC St Radboud, Nijmegen, The Netherlands.

${ }^{4}$ Department of Urology, Arhus University Hospital, Arhus, Denmark.

${ }^{5}$ Department of Urology, AUSL Modena, Italy.

${ }^{6}$ Department of Radiology, Medical University Innsbruck, Innsbruck, Austria.

${ }^{7}$ Department of Urology, Sunderland Royal University Hospital, Sunderland, United Kingdom.

${ }^{8}$ Department of Radiology, Massachusetts General Hospital, Harvard Medical School, Boston, Massachusetts.

${ }^{9}$ Department of Radiation Oncology, Leuven Cancer Institute, UZ Gasthuisberg, Leuven, Belgium.

${ }^{10}$ Department of Urology, Rheinisch-Westfalische Technische Hochschule (RWTH), Aachen, Germany.

${ }^{11}$ Interdisciplinary Brachytherapy Unit, University of Lubeck, Lubeck, Germany.

${ }^{12}$ Section of Oncology and Palliative Medicine, School of Medicine, Cardiff University, Cardiff, United Kingdom.

${ }^{13}$ Section of Pathological Anatomy, Polytechnic University of the Marche Region, School of Medicine, United Hospitals, Ancona, Italy.

${ }^{14}$ Division of Urologic Surgery, Duke University Medical Center, Durham, North Carolina.

${ }^{15}$ Division of Urologic Oncology, Department of Urology, New York University, Langone Medical Center, New York, New York.

${ }^{16}$ Department of Urology, Harlaching Klinikum-Munich, Munich, Germany.

${ }^{17}$ Department of Urology, Cliniques Universitaires Saint-Luc, Brussels, Belgium.

${ }^{18}$ Department of Urology, Princess Margaret Hospital, Toronto, Ontario, Canada.
} 


\section{Introduction}

D URING THE PAST TWO DECADES, there has been a trend toward earlier diagnosis of prostate cancer that stems from greater public and professional awareness, leading to the adoption of both formal and informal screening strategies. ${ }^{1}$ The effect of this has been to identify men with smaller tumors at an earlier stage that occupy only $5 \%$ to $10 \%$ of the prostate volume, with a greater propensity for unifocal or unilateral disease. ${ }^{2-4}$ This observation has led several groups to explore whether the prevailing strategy that has been adopted for the surgical management of all other solid organ cancers could be applied to the prostate. The strategy is one of organ preservation.

Urologists will recall the rather controversial minirevolution that partial nephrectomy induced. It did not take long to replace radical nephrectomy as the treatment of choice for a large number of patients with small tumors even in the presence of a normal contralateral kidney. The same type of revolution appears to be happening in prostate cancer treatment. In 2007, three groups proposed that nondiseased prostate tissue be left untreated in both the hope and expectation that, as a result, genitourinary function might be preserved and the tumor treated adequately. ${ }^{5-7}$

Since these initial position articles. some investigators and several clinicians have started to treat patients in a way that preserves prostate tissue. This type of treatment has become known as focal therapy. As a result, a number of approaches have emerged, with each incorporating a diverse spectrum of case selection algorithms, varying definitions of what is meant by focal therapy, a number of energy sources, and a divergent view of what is deemed a successful outcome. ${ }^{4,8}$
To identify areas in which agreement could be derived, a 1-day consensus meeting was organized. This article summarizes the conduct and output of that meeting.

\section{Materials and Methods}

The consensus meeting was held on June 13, 2009, at the end of the 2nd International Workshop on Focal Therapy and Imaging in Prostate and Kidney Cancer (Amsterdam, The Netherlands: www.focaltherapy.org). The contributors comprised a multidisciplinary and international panel of experts and were selected on the basis of their expertise in any one or more of the topics on the agenda for discussion. There were representation and endorsement from the European Society for Therapeutic Radiology and Oncology (ESTRO), the European Organization for Research and Treatment of Cancer (EORTC-GU), the European Association of Urology (EAU) guidelines committee on prostate cancer, the EAU Section of Urological Technology (ESUT), the Society of Urologic Oncology (SUO) and the Endourological Society. The attributes and affiliations of the contributors are described in Table 1.

The conduct of the meeting conformed to an informal consensus process in that no formal scoring system was used to measure the level of agreement that existed before or after the meeting. ${ }^{9}$ The process, however, did conform to the three generally accepted stages of a consensus process. ${ }^{10}$ Items for discussion were preselected and discussed by individual members before the meeting and allocated a specific time during the meeting. During the meeting, a brief presentation was made by an invited expert (scene setting). This was used

Table 1. Attributes and Affiliations of the Contributors

\begin{tabular}{|c|c|c|c|c|}
\hline Name & Specialty & Affiliation & Expertise & Country \\
\hline J. de la Rosette & Urology & Endourological Society & Uro-oncology & Netherlands \\
\hline H. Ahmed & Urology & & Uro-oncology & United Kingdom \\
\hline J. Barentsz & Radiology & & GU imaging (MRI) & Netherlands \\
\hline T. Bjerklund Johansen & Urology & & Uro-oncology & Denmark \\
\hline M. Brausi & Urology & EORTC-GU & Uro-oncology & Italy \\
\hline M. Emberton & Urology & EAU-ESUT & Uro-oncology & United Kingdom \\
\hline F. Frauscher & Radiology & EAU-ESUI & GU imaging (ultrasound) & Austria \\
\hline D. Greene & Urology & & Uro-oncology & United Kingdom \\
\hline M. Harisinghani & Radiology & & GU imaging (MRI) & United States \\
\hline K.Haustermans & $\begin{array}{l}\text { Radiation } \\
\text { oncology }\end{array}$ & ESTRO & $\begin{array}{l}\text { External radiation and } \\
\text { brachytherapy }\end{array}$ & Belgium \\
\hline A Heidenreich & Urology & EAU Guidelines & Uro-oncology & Germany \\
\hline G. Kovacs & $\begin{array}{l}\text { Radiation } \\
\text { oncology }\end{array}$ & ESTRO & $\begin{array}{l}\text { Certified brachytherapy } \\
\text { expert }\end{array}$ & Germany \\
\hline M. Mason & $\begin{array}{l}\text { Radiation } \\
\text { oncology }\end{array}$ & EAU guidelines & Oncology & United Kingdom \\
\hline R Montironi & Pathology & EAU & Uropathology & Italy \\
\hline V. Mouraviev & Urology & & Uro-oncology & United States \\
\hline Th.M. de Reijke & Urology & EORTC-GU & Uro-oncology & The Netherlands \\
\hline S. Taneja & Urology & SUO & Uro-oncology & United Kingdom \\
\hline S. Thuroff & Urology & EAU-ESUT & Uro-oncology & Germany \\
\hline B. Tombal & Urology & EORTC-GU & Uro-oncology & Belgium \\
\hline J. Trachtenberg & Urology & Endourological Society & Uro-oncology & Canada \\
\hline H. Wijkstra & Urology & EAU-ESUI & Imaging (ultrasound) & Netherlands \\
\hline T.J. Polascik & Urology & Endourological Society & Uro-oncology & United States \\
\hline
\end{tabular}

GU = genitourinary; MRI = magnetic resonance imaging; EORTC-GU = European Organization for Research and Treatment of CancerGenitourinary; EAU-ESUT = European Association of Urology-Section of Urological Technology; EAU-ESUI=European Association of Urology-Section of Urological Imaging; ESTRO=European Society for Therapeutic Radiology and Oncology; SUO=Society of Urologic Oncology. 
as the basis for moderated discussion (Level 1). After this discussion, any individual or group having concerns was identified by the moderator and resolution sought (Level 2). Once this had been completed a call for consensus was made (Level 3). This was achieved by a show of hands.

Contributors who were not invited to present a scene-setting preamble were aware of the items for discussion before the meeting and therefore also had the opportunity to prepare. Items selected for discussion are shown in Table 2. All contributors to the consensus process have seen and approved this manuscript and, by agreeing to authorship, concur with the contents of this article.

\section{Results}

\section{Definition of focal therapy}

The following definition was agreed: Focal therapy is a type of treatment that aims to eradicate known cancer within the prostate and at the same time preserve uninvolved prostatic tissue with the aim of preserving genitourinary function.

The discussion was mostly concentrated on the issue of "measurable disease." It was accepted that because a biopsy result was negative in one sector of the prostate, this did not necessarily imply that the sector was free of disease. The definition tries to embrace this view by use of the word "known."

The concept of "clinically important cancer" was discussed in contrast to the notion of any measurable cancer. What this concept introduces is that some low-volume cancers that are of low grade are unlikely to be of any clinical relevance to a person over a 15- to 20-year period. If this notion of "indolent" disease is accepted, then some focal therapy protagonists have argued that low-volume, low-grade cancer could quite safely be left in the untreated part of the prostate with little clinical consequence. ${ }^{3,11,12}$ While this was thought to be an important research question for the future, it was agreed that on the basis of current evidence and expert opinion, untreated tissue should be free of measurable disease.

\section{Patient selection for focal therapy}

The primary objective of treatment should be eradication of measurable disease. This objective has as its aim a reduction in the risk of a prostate-cancer-related detrimental effect on quality of life (QoL) or death. In addition, while the intent of treatment should be for a one-time therapy, patients need to be aware of the possibility of further treatment in the future.

Consensus was reached in a number of areas in relation to candidate selection for focal therapy. These are summarized in Table 3. The greatest area of contention was that of Gleason grade. A number of panel members were of the opinion that focal therapy should be regarded as an alternative to active

Table 2. Items Selected for Discussion

\begin{tabular}{ll}
\hline Item 1. & Definitions of focal therapy \\
Item 2. & Patient selection of focal therapy \\
Item 3. & Diagnostic pathway \\
Item 4. & Treatment modalities capable of focal therapy \\
Item 5. & Evaluation of outcome and follow-up \\
\hline
\end{tabular}

surveillance-men who were deemed to have low-risk, lowvolume disease that was normally suited for active surveillance could have access to a treatment that provided a form of therapy for their "cancer" with the greatest chance for preservation of genitourinary functions. This is based on the observation that these men are least likely to demonstrate adverse pathology, extraprostatic disease, or biochemical relapse after radical therapy and are, therefore, most representative of the "overtreated" prostate cancer population. ${ }^{13,14}$

It would be correct to point out that this opinion was predominantly nested in members from the United States or Canada and represented the position of the SUO. The divergent view was that men with intermediate grade, Gleason score 7, should also be included-this opinion meant that focal therapy could also be an alternative low-morbidity therapy for those men who did require treatment because of the presence of Gleason pattern 4 disease. This viewpoint recognized that a number of series and clinical trials had already recruited and treated men with nondominant Gleason pattern 4, both in Europe and North America. A consensus recommendation was reached on this point, with the panel agreeing that low-volume, nondominant Gleason pattern 4 found on template transperineal biopsies would probably not represent a high-risk strategy for focal therapy and should be permissible. Any Gleason pattern 4 found on standard transrectal biopsies, however, should be regarded with caution, although the panel accepted that a strict recommendation to exclude this would not be consistent with many current focal therapy protocols.

\section{Assessment of patients who may be candidates for focal therapy}

It was agreed that current transrectal ultrasonography (TRUS) biopsy regimens are inadequate for the purposes of candidate selection for focal therapy. The reason for this is that the random and systematic error associated with TRUSguided biopsy was overcome, in the past, by treating the whole prostate with either surgery or with radiation. It was agreed that in using a whole-gland approach, the characterization of the cancer was less important than it is for the therapies of active surveillance and focal therapy. Active surveillance imposes the additional burden of accurate risk stratification, because few clinicians would advise this form of treatment to men with moderate- or high-risk disease. The process of repeated biopsy addresses this to some degree. ${ }^{15}$ Many agree that TRUS biopsy is still too inaccurate for this purpose, however. To this burden of accurate risk stratification, the clinician who is advising the patient of his suitability for focal therapy has the additional task of defining the topography of the disease-in other words, its location and extent. ${ }^{16}$ In a sense, the focal therapist is more concerned with the limits of where disease is absent to a high level of certainty. TRUS-guided biopsy serves this interest poorly. ${ }^{17}$

There existed unanimous agreement that the current gold standard for characterizing men who are considering focal therapy is transperineal prostate biopsy using a templateguided approach. ${ }^{18,19}$ When used with a 5-mm sampling frame, this approach can rule in and rule out prostate cancer foci of $0.5 \mathrm{cc}$ and $0.2 \mathrm{cc}$ volume with $90 \%$ certainty. ${ }^{20}$

It was accepted that this approach is not yet widely available and carried a high burden on healthcare services because 
1. Candidates for focal therapy should ideally undergo transperineal template mapping biopsies, although a state-of-the-art multifunctional MRI with TRUS biopsy at expert centers may be acceptable.

2. Candidates for focal therapy should have a life expectancy of 10 or more years.

3. Patients with previous prostate surgery should be counseled with caution.

4. Patients with previous radiotherapy to the prostate or pelvis should not be treated until more data are available, although the panel accepted that focal salvage therapy may be a possibility in the future.

5. The effects of focal therapy on men with lower urinary tract symptoms are not well known. These men should be counseled with caution.

6. There will be specific attributes that are more related to the energy source than to focal therapy in general. Issues such as prostate size, presence of prostatic calcification, cysts, TUR cavity, access to rectum, and concurrent inflammation of rectal mucosa may need to be taken into consideration when selecting the optimal therapy.

7. Focal therapy should be limited to patients of low to moderate risk

8. Focal therapy should be limited to men with clinical $\mathrm{T}_{2 \mathrm{a}}$ or less $\mathrm{N}_{0} \mathrm{M}_{0}$ disease

9. Focal therapy should be limited to men with radiologic $\leq \mathrm{T}_{2 \mathrm{~b}} \mathrm{~N}_{0} \mathrm{M}^{0}$ disease

10. Defining the topography of the cancer is important. Disease that is predominantly apical or anterior in disposition may be technically difficult to manage with existing treatment modalities.

11. The long-term effects of focal therapy on potency/erectile functions are not known. Men should be counseled in this regard before therapy

$\mathrm{MRI}=$ magnetic resonance imaging; TRUS $=$ transrectal ultrasonography; TUR $=$ transurethral resection.

of its requirement for anesthesia, pathology processing, and reporting time.

The contributors to the consensus panel were convinced that imaging was going to play an important role in selecting patients as suitable candidates in the future. ${ }^{21}$ The contributors were less convinced of the current role of imaging in the diagnostic pathway for these men. ${ }^{22}$ There was overall agreement that multiparametric functional MRI and new TRUS techniques show promising results in multiple centers and publications in relation to characterizing men with prostate cancer and localizing their tumor. Based on these results, it is expected that these techniques will play a major role in the future for the selection, performance, and follow-up of patients with focal therapy. Therefore, multicenter studies need to be urgently prioritized. ${ }^{23-25}$

\section{Treatment modalities for focal therapy}

The consensus panel was in agreement that traditional therapies did not have the attributes to treat in a focal manner. Surgery and external beam radiation therapy were not yet ready for use in focal treatment. Most focal therapies to date have been achieved with ablative technologies: Cryotherapy, high intensity focused ultrasound (HIFU), or photodynamic therapy. At present, focal therapy using cryosurgery has the most experience. ${ }^{26}$ The published data relating to these technologies are presented and discussed in recent reviews and a number of prospective single-center trials are under way. ${ }^{27}$ Most recently, the first report of the use of thermal laser for focal therapy has been published. ${ }^{28}$

The role of radiotherapeutic approaches is at present less clear. Possible options, however, include high-dose-rate brachytherapy, low-dose-rate seed implantation brachytherapy, intensity modulated, hypofractionated photon irradiation, and proton beam therapy. It was agreed by the group, however, that none of these had been tried in the primary focal therapy arena. Experimental technologies, such as microwave, electroporation, and radiofrequency interstitial tumor ablation and magnetic nanoparticle thermotherapy are also currently under investigation in early clinical trials. All of the above have the attributes that can, at least from a technical perspective, treat a specific area or one lobe of the prostate with little if any effect on the contralateral lobe.

\section{Evaluation of outcome and follow-up}

There are three main aspects to evaluating the outcome of focal therapy: The assessment of functional status, the oncologic efficacy, and the impact on subsequent therapy.

The first key outcome domain is functional status that, in turn, relates to disease-specific and generic QoL. ${ }^{29}$ The panel agreed that case series had demonstrated that focal therapy could achieve significantly lower toxicity rates with preservation of genitourinary function approaching $80 \%$ to $90 \%$ after focal cryosurgery or HIFU. ${ }^{30}$ All but a few of these outcomes, however, were patient reported on validated questionnaires. There are no validated instruments that have been designed for use in men who are undergoing focal therapy. As a result, the consensus panel has recommended that existing self-reported measures of health status should be used in order to compare outcome to other treatment modalities. The scales in current use that would assess the components of functional status include the following: International Prostate Symptom Score, IIEF, International Continence Society scale of urinary continence, Functional Assessment of Cancer Therapy-Prostate, EuroQoL, Short Form-36, and EORTC QoL.

The panel was in agreement that determining oncologic efficacy at this stage of the evolution of the technology was best achieved by an interval post-treatment biopsy of the treated part of the prostate. Given that the panel recommended that patients are initially characterized by transperineal template-guided biopsies, it seemed to the panel sensible that the same test is used to assess absence of cancer. The panel does accept, however, that this will pose a significant burden on both the patient and the healthcare system, and thus, at this time, a TRUS-based office biopsy may be a reasonable alternative. It was the view of the panel that posttreatment evaluation by biopsy should take place between 6 and 12 months after treatment because such a time interval 
would allow for resolution of inflammatory changes and establishment of stable scar tissue.

In the future, it may be that imaging, particularly with multi-functional MRI, or contrast-enhanced ultrasonography may be useful in reducing the burden of post-treatment biopsies, although this needs further evaluation before any recommendation is made. ${ }^{19,28,31,32}$

Biomarkers in the interval between biopsy evaluations will play an important role, although the panels' view was that the prostate-specific antigen (PSA) kinetics of partially treated prostate tissue are largely unknown. The panel felt that PSA should be measured at least as much as is common practice for standard therapy. As such, the panel recommended PSA measurements at 3-monthly intervals for the first year after treatment and every 6 months thereafter. Long-term followup, including biomarkers, biopsies, and imaging, is important to understand the effect on the treated side and the natural history of the untreated tissue.

The role of other biomarkers, such as PCA3, is at present not well defined. They may prove to be useful in monitoring the nontreated region. The panel strongly recommended the collection of urine, serum, and tissue for archiving so that future interrogation of such archives can be used to develop biomarkers that can be linked to long-term outcomes after focal therapy.

The third key outcome domain relates to the impact of focal therapy on subsequent treatments. The panel acknowledged that primary treatment fails in a number of men irrespective of the kind of treatment they were allocated. The effect of focal therapy on the likely salvage procedures of surgery and radiotherapy remains unknown. The panel was unanimous that this aspect of focal therapy be monitored closely so that men are not placed at undue risk if their primary therapy was to fail.

\section{Conclusion}

The consensus process derived considerable agreement in all items tabled. Where no agreement was possible, the inevitable resolution was the recommendation of further research, because in nearly all instances, disagreement was because of clinical uncertainty and not divergent clinical opinion.

While most of the recommendations that resulted remain uncontroversial, the reader should interpret our results in light of certain methodologic limitations. The first of these relates to the composition of the group. The participants were selected for their expertise in focal therapy and were therefore by default enthusiasts. There are plenty of skeptics who, had they been present, would have voted in different ways. As a result, the recommendations may not be representative of the opinion of the wider urologic community and may therefore be difficult to generalize beyond those interested in undertaking or evaluating this novel approach.

The second reasonable criticism may lie with our approach. We chose, for reasons of convenience, an informal consensus process in which no quantitative measure of agreement was derived. Participants, at the time of deriving consensus, were required to vote for or against. If the vote was close to or unanimous, the vote was carried. If the vote was split, it was deemed that agreement was not possible. The bias that can result from such an approach is that vociferous and forceful personalities can exert too much influence in the overall scoring. While we accept this criticism, this effect was mitigated by the presence of two chairpersons and introduction of topics by different invited experts. Before closure of any topic, views were invited from all those present.

Despite these methodologic limitations, we believe that the consensus process was helpful in refining some of the conceptual and practical considerations that relate to focal therapy for men with localized prostate cancer. The participation in this meeting and endorsement of the consensus report by representatives from the ESTRO, EORTC, and the EAU guidelines committee on prostate cancer, as well as ESUT, SUO, and the Endourological Society signify its position and carries additional weight to the outcomes. Obviously, this is a field that will progress rapidly. Future meetings such as this will have the benefit of including data that are derived from phase II clinical trials on which further recommendations can be made.

\section{Acknowledgments}

Mark Emberton is funded by UCLH/UCL Comprehensive Biomedical Research Centre, London, United Kingdom; Hashim Ahmed holds a Medical Research Council UK Fellowship.

\section{Disclosure Statement}

No competing financial interests exist.

\section{References}

1. Schröder FH, Hugosson J, Roobol MJ, et al. ERSPC Investigators. Screening and prostate-cancer mortality in a randomized European study. N Engl J Med 2009;360:13201328.

2. Mouraviev V, Mayes JM, Polascik TJ. Pathologic basis of focal therapy for early-stage prostate cancer. Nat Rev Urol 2009;6:205-215.

3. Cooperberg MR, Broering JM, Kantoff PW, Carroll PR. Contemporary trends in low risk prostate cancer: Risk assessment and treatment. J Urol 2007;178:S14-S19.

4. Polascik TJ, Mayes JM, Sun L, et al. Pathologic stage T2a and $\mathrm{T} 2 \mathrm{~b}$ prostate cancer in the recent prostate-specific antigen era: Implications for unilateral ablative therapy. Prostate 2008;68:1380-1386.

5. Ahmed HU, Pendse D, Illing R, et al. Will focal therapy become a standard of care for men with localized prostate cancer? Nat Clin Pract Oncol 2007;4:632-642.

6. Eggener SE, Scardino PT, Carroll PR, et al. International Task Force on Prostate Cancer and the Focal Lesion Paradigm. Focal therapy for localized prostate cancer: A critical appraisal of rationale and modalities. J Urol 2007;178:22602267.

7. Crawford ED, Barqawi A. Targeted focal therapy: A minimally invasive ablation technique for early prostate cancer. Oncology (Williston Park) 2007;21:27-34.

8. De la Rosette JJ, Mouraviev V, Polascik TJ. Focal targeted therapy will be a future treatment modality for early stage prostate cancer. Eur Urol 2009;8(suppl):424.

9. Fink A, Kosecoff J, Chassin M, Brook RH. Consensus methods: Characteristics and guidelines for use. Am J Public Health 1984;74:979-983.

10. $\overline{\text { Consensus.net. }}$ 
11. Ahmed HU, Emberton M. Active surveillance and radical therapy in prostate cancer: Can focal therapy offer the middle way? World J Urol 2008;26:457-467.

12. Scardino PT. Focal therapy for prostate cancer. Nat Rev Urol 2009;6:175.

13. Mouraviev V, Sun L, Madden JF, et al. Prostate cancer laterality does not predict prostate-specific antigen recurrence after radical prostatectomy. Urology 2007;70:1141-1145.

14. Tareen B, Godoy G, Sankin A, et al. Laterality alone should not drive selection of candidates for hemi-ablative focal therapy. J Urol 2009;181:1082-1090.

15. De la Rosette JJ, Wink MH, Mamoulakis C, et al. Optimizing prostate cancer detection: 8 versus 12 -core biopsy protocol. J Urol 2009;182:1329-1336.

16. Polascik TJ, Mayes JM, Schroeck FR, et al. Patient selection for hemiablative focal therapy of prostate cancer: Variables predictive of tumor unilaterality based upon radical prostatectomy. Cancer 2009;115:2104-2110.

17. Tareen B, Godoy G, Sankin A. Can contemporary transrectal prostate biopsy accurately select candidates for hemi-ablative focal therapy of prostate cancer? BJU Int 2009;104:195-199.

18. Onik G, Miessau M, Bostwick DG. Three-dimensional prostate mapping biopsy has a potentially significant impact on prostate cancer management. I Clin Oncol 2009;27:43214326.

19. Onik G, Barzell W. Transperineal 3D mapping biopsy of the prostate: An essential tool in selecting patients for focal prostate cancer therapy. Urol Oncol 2008;26:506-510.

20. Crawford ED, Wilson SS, Torkko KC, et al. Clinical staging of prostate cancer: A computer-simulated study of transperineal prostate biopsy. BJU Int 2005;96:999-1004.

21. Wink M, Frauscher F, Cosgrove D, et al. Contrast-enhanced ultrasound and prostate cancer; a multicentre European research coordination project. Eur Urol 2008;54:982-992.

22. Kelloff GJ, Choyke P, Coffey DS; Prostate Cancer Imaging Working Group. Challenges in clinical prostate cancer: Role of imaging. AJR Am J Roentgenol 2009;192:1455-1470.

23. Ahmed HU, Kirkham A, Arya M, et al. Is it time to consider a role for MRI before prostate biopsy. Nat Rev Clin Oncol 2009;6:197-206.

24. Turkbey B, Pinto PA, Choyke PL. Imaging techniques for prostate cancer: Implications for focal therapy. Nat Rev Urol 2009;6:191-203.

25. Villers A, Puech P, Mouton D, et al. Dynamic contrast enhanced, pelvic phased array magnetic resonance imaging of localized prostate cancer for predicting tumor volume: Correlation with radical prostatectomy findings. J Urol 2006; 176:2432-2437.
26. Polascik TJ, Mouraviev V. Focal therapy for prostate cancer. Curr Opin Urol 2008;18:269-274.

27. Ahmed HU, Moore C, Emberton M. Minimally-invasive technologies in uro-oncology: The role of cryotherapy, HIFU and photodynamic therapy in whole gland and focal therapy of localised prostate cancer. Surg Oncol 2009;18:219-232.

28. Lindner U, Weersink RA, Haider MA, et al. Image guided photothermal focal therapy for localized prostate cancer: Phase I trial. J Urol 2009;182:1371-1377.

29. Sanda MG, Dunn RL, Michalski J, et al. Quality of life and satisfaction with outcome among prostate-cancer survivors. N Engl J Med 2008;358:1250-1261.

30. Onik G, Vaughan D, Lotenfoe R, et al. The "male lumpectomy": Focal therapy for prostate cancer using cryoablation results in 48 patients with a least 2-year follow-up. Urol Oncol 2008;26:500-505.

31. Kirkham AP, Emberton M, Hoh IM, et al. MR imaging of prostate after treatment with high-intensity focused ultrasound. Radiology 2008;246:833-844.

32. Rouvière $\mathrm{O}$, Girouin N, Glas L, et al. Prostate cancer transrectal HIFU ablation: Detection of local recurrences using T2-weighted and dynamic contrast-enhanced MRI. Eur Radiol 2009;Aug 19. E-pub ahead of print.

Address correspondence to:

J. de la Rosette, M.D.

Department of Urology G4-105 AMC University Hospital Amsterdam

The Netherlands

E-mail: j.j.delarosette@amc.uva.nl

Abbreviations Used
EAU $=$ European Association of Urology
EORTC-GU $=\begin{gathered}\text { European Organization for Research } \\ \text { and Treatment of Cancer-Genitourinary }\end{gathered}$
ESTRO $=$ European Society for Therapeutic Radiology
$\quad$ and Oncology
ESUT $=$ EAU Section of Urological Technology
GU $=$ genitourinary
HIFU $=$ high intensity focused ultrasound
MRI $=$ magnetic resonance imaging
PSA $=$ prostate-specific antigen
QoL $=$ quality of life
SUO $=$ Society of Urologic Oncology
TRUS $=$ transrectal ultrasonography


This article has been cited by:

1. Vladimir Mouraviev, Truls Erik Bjerklund Johansen, Thomas J. Polascik . 2010. Contemporary Results of Focal Therapy for Prostate Cancer Using CryoablationContemporary Results of Focal Therapy for Prostate Cancer Using Cryoablation. Journal of Endourology 24:5, 827-834. [Abstract] [Full Text] [PDF] [PDF Plus]

2. Jean de la Rosette, Inderbir Gill, Thomas Polascik . 2010. Focal Therapy for Prostate and Renal Cancer-Are We Ready for It?Focal Therapy for Prostate and Renal Cancer-Are We Ready for It?. Journal of Endourology 24:5, 663-663. [Citation] [Full Text] [PDF] [PDF Plus] 